\title{
Estimation of Nonalcoholic Fatty Liver Disease in Patients with Normal BMI on Ultrasound
}

\author{
Umme Habiba* \\ Doctor of Medical Imaging Technology, Department of Allied Health Sciences, Superior College Lahore, \\ University Campus, 17-KM Raiwaind Road, Kot Arain, Lahore, Pakistan \\ Laraib Afzal \\ Doctor of Medical Imaging Technology, Department of Allied Health Sciences, Superior College Lahore, \\ University Campus, 17-KM Raiwaind Road, Kot Arain, Lahore, Pakistan \\ Waqas Ahmad \\ Doctor of Medical Imaging Technology, Department of Allied Health Sciences, Superior College Lahore, \\ University Campus, 17-KM Raiwaind Road, Kot Arain, Lahore, Pakistan
}

Uttroba Shahid

Masters in Diagnostics Ultrasound, Lecturer, Department of Allied Health Sciences, Superior College Lahore, University Campus, 17-KM Raiwaind Road, Kot Arain, Lahore, Pakistan

\author{
Mansoor Bkhtiary \\ MBBS, MCPS, DMRD \\ Radiologists in DHA Medical Centre, Lahore.
}

Rana Muhammad Athar Azeem Shams

Masters in Medical Imaging Technology, Lecturer, Department of Allied Health Sciences, Superior College Lahore, University Campus, 17-KM Raiwaind Road, Kot Arain, Lahore, Pakistan

Rana Muhammad Bakhtawar Khan Sajawal

Masters in Mathematics, Lecturer, Department of Allied Health Sciences, Superior College Lahore, University Campus, 17-KM Raiwaind Road, Kot Arain, Lahore, Pakistan

Amna Babar

Masters in Diagnostic Ultrasound, Lecturer, Department of Allied Health Sciences, Superior College Lahore, University Campus, 17-KM Raiwaind Road, Kot Arain, Lahore, Pakistan

The research is financed by Asian Development Bank. No. 2006-A171(Sponsoring information)

\section{Abstract}

Background: Non-Alcoholic fatty liver disease is common in adults and it is increasing in patients with normal BMI in Asian countries. Non-alcoholic fatty liver disease (NAFLD) occurs not only in obese individuals but also in non-obese ones. The association between NAFLD and metabolic events in a non-obese population is also evident..

Objective: To estimate nonalcoholic fatty liver disease in patients with normal BMI on ultrasound.

Methodology: Analytical Cross-sectional prospective study in which 59 patients were enrolled in the research. All the patient's data had been composed from indoor of hospital, outdoor of hospital, DHA Medical Center, Lahore. After well-versed consent, data was composed through ultrasound machine. The data, such as patient characteristics, hypertension, impaired fasting glucose, were extracted from medical records, and statistical analysis was performed.

Results: The present study is retrospective cross sectional observational study.60 patients (29males $49.2 \% 31$ female $50.8 \%$ ) were enrolled in this study. According to abdominal ultrasonography, $72.9 \%$ of patients with normal BMI were diagnosed to have Non-alcoholic fatty liver disease and identified to have fatty changes in the liver.

Conclusion: In our study we estimated that nonalcoholic fatty liver disease was present in patients with normal body mass index by imaging the echotexture of liver on ultrasound. Having increased echogenicity, due to poor diet and other associated diseases such as high blood pressure, impaired fasting glucose and low HDL cholesterol patients were getting NAFLD.

Keywords: Nonalcoholic fatty liver disease (NAFLD), Body Mass Index (BMI), Ultrasonography (USG).

DOI: $10.7176 / \mathrm{JHMN} / 92-03$

Publication date:August $31^{\text {st }} 2021$ 


\section{Introduction}

A chronic liver disease that has showed progress in cirrhosis and hepatocellular cancer is nonalcoholic fatty liver disorder (NAFLD). NAFLD seems closely associated with obesity and insulin resistance, including diabetes, and other metabolic syndrome characteristics, such as high triglycerides and low HDL.

The estimated prevalence in the general population ranges from $3 \%$ to $24 \%$, with the most estimates ranging from $6 \%$ to $14 \%$. Some data imply that Mexican Americans are more likely than non-Hispanic whites to have NAFLD while blacks are less prevalent. The advanced phases of NAFLD include old age, greater levels of body mass, diabetes, high blood pressure, high triglycerides and/or resistance to insulin. More severe illness can also be shown by an AST/ALT ratio of more $>1 .{ }^{[1]}$ In the past decade, the antagonism to insulin has been viewed as the main hidden weapon of pathophysiology despite its absence in fully influencing people. A significant pathophysiological component is NAFLD which is particularly weight-driven insulin opposition. Fiery angles are also of pivitol importance especially in fibrosis associated with NASH and NASH, usually resulting in an overpowering fire in the liver. ${ }^{[2]}$ Heaviness, type 2 diabetes, metabolism, including dyslipidemias and hypertension is the most important risk component of NAFLD. Hepatic fat can be associated with infections other than the metabolic conditions, which could lead to the differential conclusion of a common fatty liver disease. Because of the evident amount of fatty tissue in lean patients, i.e. BMI da $25.0 \mathrm{~kg} / \mathrm{m} 2$, there may be contrasts among sleek and contrasting NAFLD disease components with heavy patients. ${ }^{[3]}$ Liver is a sequestered, reddish earthy structure with two flaps of varying form and size. Human liver generally takes approximately 1,5 kilograms $(3.3 \mathrm{lb})$ and is approximately $15 \mathrm{~cm}$ wide $(6 \mathrm{in})$. Specific reference limit is $971-1,861 \mathrm{~g}(2,13-4,11$ pound) for males and $600-1,771 \mathrm{~g}$ for ladies (1,31-3.90 pound) of men. The size varies substantially across men. Either the heaviest structure within human humans and the largest organ. It lies underneath the stomach on one side of the stomach and surmounts the gallic bladder in the right upper region of the stomach depression. ${ }^{[4]}$ When viewed from a higher place - a pre-requisite and a left flap - the liver is brutally divided into two halves and the quadrilateral parts are observed underneath all liver lobes. In the middle area of the hepatic hilum, the opening known as the porta hepatis is integrated, which provides the standard bile channel and the base liver duct. On condition that these parts are useful, the ductal, vein and course division into left and right branches and the regions of the hepatic make protrusions of the left and right side. ${ }^{[5]}$ It takes a role in the production, multiplication and development of chemical digestion and process transporting proteins. Finally, cups and primary cells play an important role in the resistive structure of the body. Bile is a major liquid since it releases a product not released by excretory systems and helps with phospholipid intake and treatment by emitting salts and acids from digestive juices. The bile comes from keratinocytes and, although the various chemicals are not present, mainly consisting of water, electrodialyzes, steroids, bile acids, cholesterol, bile pigment, bilirubin and phospholipids. ${ }^{[6]}$ The liver is considered to be normal in ultrasound if the echotexture is uniform with no acoustic restriction; the portable veins are Obvious, stomach is extremely visual and the echogenicity of the renal parenchyma can be compared to or marginally higher. The liver is described as fatty liver if the liver has zones of echogenicity that are substantially increased, comparative to renal parenchyma, the stomach ultrasonography bar is constrained or the portal vein echo-dividers are less visible. ${ }^{[7]}$ As a metric to quantify obesity the body mass index BMI is $>30 \mathrm{~kg} / \mathrm{ms} 2$. Many patients without obesity (up to $25 \%$ ) suffer from NASFLD, leading to cardiovascular disease and diabetes in Asians among patients with normal BMI. It is vital for patients with NAFLD to be investigated for metabolic syndrome and to have detrimental outcomes with respect to the normal BMI. ${ }^{[8]}$

\subsection{Material and Methods}

This is an analytical cross-sectional study. The data was collected from DHA Medical Centre, Lahore. the duration of data study was from January to May 2021. Total 59 subjects were enrolled is this which completed the inclusion criteria of this study. Patients having normal BMI $18-25 \mathrm{~kg} / \mathrm{ms}^{2}$ and above 18 years were included. Patients having Hepatitis B and C, iron deficiency and who consumed alcohol are excluded. Ultrasound machine, Apollo, was used with convex probe of 3-5 MHz Body mass index was calculated by patient's height and weight. After calculation transabdominal scan was performed. Liver parenchyma, echogenicity and fatty appearance was observed. Afterwards, patients' blood pressure was measured by using sphygmomanometer. 120/80 is normal parameter above this level it is declared as high BP. Blood glucose was measured when patient was in fasting state. 110 is normal measurement above and below this level was labelled as impaired fasting glucose. Other variables like regular exercise, low HDL cholesterol and smoking was taken from patient history and from medical records. Body fat percentage was calculated from BMI and Age of the patients. SPSS Computer software, version 25 was used to analysis the data. The rules and regulations set by the ethical committee of The Superior College were followed while conducting the research and the rights of the research participants were respected. Written informed consent form attached was taken from all the participants.

\subsubsection{Results:}

A total of 59 patients in which 29 were males and 30 were females with Nonalcoholic fatty liver disease having 
normal BMI. The mean patients were 39.17 and 12.34 years old on average. $72.9 \%$ of patients having increased echogenicity was labeled as nonalcoholic fatty liver disease. Below are graphs of the average of the echotexture, impaired fasting glucose, low HDL cholesterol, regular exercise, smoking and body fat percentage.

\subsubsection{Discussion:}

A research conducted in Bangladesh in 2020 by M Masudur Rahman, et al aims to determine the generality of non-hefty corpulent NAFLD, consider the clinical and metabolic sociodemographic attributes between nonstopping and fat NAFLD subjects and determine the hazardous components of the NAFLD. It had $18.5 \%$ of its members, one quarter of them were bodyless. The metabolic profile of the sizeful and unheavy NAFLD was equivalent in addition to the weight list. The nonalcoholic fatty liver disease and its deleterious effects should be evaluated and monitored by general welfare measures. In 57/804 (7.1\%) of the non-hefty and 185/501(36.93\%) of the overweight members, NAFLD was recognised. Of the magic topics, NAFLD had 24/592 (4.1\%). Fiftyseven $(23.55 \%)$ of NAFLDs had non-stop, with alanine amino-transferase elevated by five (22\%) individuals. The risk variables for NAFLD have been established during multivariate analysis, age $>40$ years, male sex, metabolism (MS), diabetes mellitus (DM), stomach corpulence, high blood pressure, dyslipidemia and heftiness. Ren-Nan Feng et al. (2014) In China, analyse NAFLD's high. NAFLD patients were more seasoned, more BMIcontaminated, with abdominal periphery, pulse, fasting blood glucose and insulin. Although lean-NAFLD patients had a reduced BMI and intersectional situation, the instinctive adiposity record was substantially greater than that of overweight body checks. Patients with Lean-NAFLD had overweight fatty oil, cholesterol for NAFLD patients with a low thickness of lipoprotein.

Hyperlipidemia, hypertension, diabetes and metabolic disease were endangered by all NAFLD patients (MetS). The overweight NAFLD (only OOR for MetS was significant: OR=1.89; 95\% CI:1.29-2.77) was more clearly associated to diabetes $(\mathrm{OR}=2.47 ; 95 \% \mathrm{CI}$ : $1.14-5.35)$, hypertension (or $=1.72 ; 95 \% \mathrm{CI}: 1.00-2.96)$ and MetS (OR+=3.19; and 95\% CI: 1.17-4.05). NFLD patients were linked to focal stoutness, notably in lean assemblages $(\mathrm{OR}=1.97,95$ percent $\mathrm{CI}: 1.38-2.80)$, or $\mathrm{OR}=2.17,95$ percent $\mathrm{C} \mathrm{I}: 1.17-4.05)$.

Our study was very much similar with their study, in our study we have discussed mainly the clinical representation of NAFLD in patients with normal BMI on ultrasound. We estimated that patients with normal BMI were likely to suffer from NAFLD due to their lack of regular exercise and poor diet routine which led them to other diseases like high blood pressure, diabetes mellitus etc. In our study aggregate of 59 patients (29male $-49.2 \%$ and 30 female-50.8\%). were designated. 43 patients with normal BMI $18-25 \mathrm{kgms}^{2}$ from total population have increased liver echogenicity seen on ultrasound. And these patients were also associated with impaired fasting glucose and high blood pressure. It is concluded from the study that $62.7 \%$ of people have low HDL cholesterol less than 40 and body fat percentage were calculated according to age and BMI of patient. Data were selected from individuals who were sent for abdominal USG. Ultrasonographic investigation was done on patients with normal BMI and then other factors like impaired fasting glucose, low HDL cholesterol, high blood pressure, regular exercise and body fat percentage were also investigated.

\section{References}

1. Clark, Jeanne M MD, MPH. the epidemiology of nonalcoholic fatty liver disease in adults. The journal of gastroenterology.2006. vol:40 pg: S5-S10.

2. Rotonya M. Carr, MD, Amanke Oranu, MD, and Vandana Khungar, MD, MSc. "Non-alcoholic fatty liver disease: Pathophysiology and management".Gastroenterol Clin North Am. 2016 Dec; 45(4): 639-652.

3. Tilg H, Hotamisligil GS: Nonalcoholic fatty liver disease: cytokine-adipokine interplay and regulation of insulin resistance. Gastroenterology 2006;131:934-945.

4. Elias, H.; Bengelsdorf, H. "The Structure of the Liver in Vertebrates". Cells Tissues Organs. 14 (4): $297-$ 337.

5. Abdel-Misih, Sherif R.Z.; Bloomston, Mark. Liver Anatomy Surgical Clinics of North America.2010 90 (4): 643-653

6. Molina, D. Kimberley; DiMaio, Vincent J.M. "Normal Organ Weights in Men". The American Journal of Forensic Medicine and Pathology. 201233 (4): 368-372.

7. Vincent Braunersreuther, Giorgio L. V., François M., and Fabrizio M. Role of cytokines and chemokines in non-alcoholic fatty liver disease. World J Gastroenterol 2012 Feb 28; 18(8): 727-735.

8. Mangesh R Pagadala, Arthur J Mc. Nonalcoholic fatty liver disease and obesity: not all about body mass index". American Journal of Gastroenterology 107(12), 1859-1861,2012

9. Joo Hee Kwak, Dae WonJun, Seung MinLee, Yong KyunCho, Yong KyunCho, Hang LakLee, Oh YoungLee, Ho Soon Choi, Byung Chul Yoon. Lifestyle predictors of obese and non-obese patients with nonalcoholic fatty liver disease: A cross-sectional study.2018 37(5), Pg:1550-1557.

10. Mitra Ahadi, Kasra Molooghi, Negin Masoudifar, Ali Beheshti Namdar, Hassan Vossoughinia, Mohammadreza Farzanehfar A review of non-alcoholic fatty liver disease in non-obese and lean individuals, PMID: 3321705 
Statistics

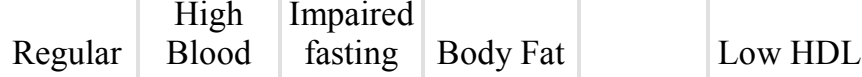

Age(Years)Gender BMI Exercise Pressure glucose PercentageSmoking CholesterolEchotexture

\begin{tabular}{|c|c|c|c|c|c|c|c|c|c|c|c|}
\hline \multirow[t]{2}{*}{$\overline{\mathrm{N}}$} & \multirow{2}{*}{ Missing } & 59 & 59 & 59 & 59 & 59 & 59 & 59 & 59 & 59 & 59 \\
\hline & & 0 & 0 & 0 & 0 & 0 & 0 & 0 & 0 & 0 & $\overline{0}$ \\
\hline \multicolumn{2}{|c|}{ Mean } & 39.17 & .49 & 1.73 & 1.47 & .69 & 1.36 & 1.54 & .51 & 1.37 & 1.73 \\
\hline \multicolumn{2}{|c|}{ Mode } & 45 & 0 & 2 & 1 & 1 & 1 & 1 & 1 & 1 & 2 \\
\hline \multicolumn{2}{|c|}{ Minimum } & 21 & 0 & 1 & 1 & 0 & 1 & 1 & 0 & 1 & 1 \\
\hline \multicolumn{2}{|c|}{ Maximum } & 72 & 1 & 3 & 2 & 1 & 2 & 3 & 1 & 2 & 2 \\
\hline
\end{tabular}

\begin{tabular}{|c|c|c|c|c|c|}
\hline \multicolumn{6}{|c|}{ Regular Exercise } \\
\hline & & Frequency & Percent & Valid Percent & Cumulative Percent \\
\hline \multirow[t]{3}{*}{ Valid } & YES & 31 & 52.5 & 52.5 & 52.5 \\
\hline & $\mathrm{NO}$ & 28 & 47.5 & 47.5 & 100.0 \\
\hline & Total & 59 & 100.0 & 100.0 & \\
\hline
\end{tabular}

\begin{tabular}{|c|c|c|c|c|c|}
\hline \multicolumn{6}{|c|}{ High Blood Pressure } \\
\hline & & Frequency & Percent & Valid Percent & Cumulative Percent \\
\hline \multirow[t]{3}{*}{ Valid } & $\mathrm{NO}$ & 18 & 30.5 & 30.5 & 30.5 \\
\hline & YES & 41 & 69.5 & 69.5 & 100.0 \\
\hline & Total & 59 & 100.0 & 100.0 & \\
\hline
\end{tabular}

\begin{tabular}{llr|r|r|r} 
& \multicolumn{2}{c}{ Impaired fasting glucose } \\
& \multicolumn{2}{c}{ Percent } & Valid Percent & Cumulative Percent \\
\hline Valid & FES & 38 & 64.4 & 64.4 & 64.4 \\
\hline & NO & 21 & 35.6 & 35.6 & 100.0 \\
\hline & Total & 59 & 100.0 & 100.0 & \\
\hline
\end{tabular}

Body Fat Percentage

\begin{tabular}{rlr|r|r|r} 
& \multicolumn{2}{c}{ Frequency } & Percent & Valid Percent & Cumulative Percent \\
\hline Valid & $20-39(8-19 \%)$ & 30 & 50.8 & 50.8 & 50.8 \\
\cline { 2 - 6 } & $40-59(11-21 \%)$ & 26 & 44.1 & 44.1 & 94.9 \\
\cline { 2 - 6 } & $60-79(13-24 \%)$ & 3 & 5.1 & 5.1 & 100.0 \\
\hline & Total & 59 & 100.0 & 100.0 & \\
\hline
\end{tabular}

\begin{tabular}{|c|c|c|c|c|c|}
\hline \multicolumn{6}{|c|}{ Smoking } \\
\hline & & Frequency & Percent & Valid Percent & Cumulative Percent \\
\hline \multirow[t]{3}{*}{ Valid } & $\mathrm{NO}$ & 29 & 49.2 & 49.2 & 49.2 \\
\hline & YES & 30 & 50.8 & 50.8 & 100.0 \\
\hline & Total & 59 & 100.0 & 100.0 & \\
\hline
\end{tabular}

Low HDL Cholesterol

\begin{tabular}{llr|r|r|r} 
& & Frequency & \multicolumn{1}{c}{ Percent } & Valid Percent & Cumulative Percent \\
\hline \multirow{2}{*}{ Valid } & Less than 40 & 37 & 62.7 & 62.7 & 62.7 \\
\cline { 2 - 6 } & Less than 60 & 22 & 37.3 & 37.3 & 100.0 \\
\cline { 2 - 6 } & Total & 59 & 100.0 & 100.0 & \\
\hline
\end{tabular}

\section{Echotexture}

\begin{tabular}{ll|r|r|r|r} 
& & Frequency & Percent & Valid Percent & \multicolumn{2}{c}{ Cumulative Percent } \\
\hline \multirow{2}{*}{ Valid } & Normal & 16 & 27.1 & 27.1 & 27.1 \\
\cline { 2 - 6 } & Increased Echogenicity & 43 & 72.9 & 72.9 & 100.0 \\
\cline { 2 - 6 } & Total & 59 & 100.0 & 100.0 & \\
\hline
\end{tabular}

Table no 1: frequency tables for age, gender BMI, regular exercise, low HDL cholesterol, impaired fasting glucose, smoking and high blood pressure. 